\section{Cureus}

Received 02/27/2014

Review began $02 / 27 / 2014$

Review ended 11/15/2014

Published 11/17/2014

\section{(c) Copyright 2014}

Kathiresan et al. This is an open access article distributed under the terms of the Creative Commons Attribution License CC-BY 3.0., which permits unrestricted use, distribution, and reproduction in any medium, provided the original author and source are credited.

\title{
Reproductive Endocrinology and Infertility Match: A Survey of the 2011-2013 Applicant Pool
}

Anupama Kathiresan ${ }^{1}$, Mousa Shamonki ${ }^{1}$, Amy Kaing ${ }^{1}$, Yenisel Cruz-Almeida ${ }^{2}$, Marcelo J. Barrionuevo $^{3}$, David I. Hoffman ${ }^{3}$, Wayne S. Maxson ${ }^{3}$, Steven J. Ory ${ }^{3}$

1. University of California, Los Angeles 2. University of Florida 3. University of Miami Miller School of Medicine

$\square$ Corresponding author: Anupama Kathiresan, anu.kathiresan@gmail.com Disclosures can be found in Additional Information at the end of the article

\section{Abstract}

Objective: The purpose of this study was to determine what objective applicant characteristics increased the likelihood of successfully matching into a Reproductive Endocrinology and Infertility (REI) fellowship position.

Methods: A prospective electronic survey was distributed to 130 applicants who participated in the 2011-2013 REI fellowship match. Applicants were asked questions regarding demographic/educational background, research experience, abstract/publication record, Council on Resident Education in Obstetrics and Gynecology (CREOG) exam scores, interview invitations, and match results. Statistical analysis was performed with Mann-Whitney U test to compare continuous and discrete ordinal variables, and Chi-square or Fisher's exact test to compare nominal variables, where appropriate.

Results: Candidates that matched were younger (30.0 vs. 32.4 years old, $p=0.02$ ); otherwise, there were no other significant differences in candidates' demographic background. Matched applicants had significantly more first author poster abstracts accepted ( $3.4 \pm 0.4 \mathrm{vs.} 1.1 \pm 0.4$, $\mathrm{p}=0.01)$. There was also a trend for matched applicants to have more publications accepted (3.3 \pm 0.8 vs. $2.0 \pm 1.0, \mathrm{p}=0.14)$ and submitted $(1.5 \pm 0.4$ vs. $0.8 \pm 0.4, \mathrm{p}=0.16)$. While candidates applied to a similar number of programs, matched applicants received more interview offers (17.1 \pm 1.1 vs. $8.7 \pm 2.1, \mathrm{p}=0.002)$, attended more interviews $(13.7 \pm 0.7$ vs. $8.1 \pm 1.8, \mathrm{p}=0.01)$, and ranked more programs $(13.3 \pm 0.7$ vs. $8.1 \pm 1.8, \mathrm{p}=0.01)$.

Conclusions: Research experience, particularly presenting poster abstracts as first author, was significantly associated with successful match into REI fellowship. There was also a trend for matched applicants to have more publications accepted and submitted at the time of the interview process.

Categories: Medical Education, Obstetrics/Gynecology

Keywords: match process, obstetrics and gynecology residency applicants, reproductive endocrinology and infertility

\section{Introduction}

The Reproductive Endocrinology and Infertility (REI) fellowship match remains one of the most competitive among obstetrics and gynecology subspecialties. Between 2008 and 2013, the 
unmatched applicant rate ranged from $32.4 \%$ to $48.6 \%$ [1]. Several factors have been speculated to influence an applicant's chances of successfully matching into a REI fellowship position including: prior research experience, publication record, and Council on Resident Education in Obstetrics and Gynecology (CREOG) exam scores. To our knowledge, no study has investigated this topic. The present study utilized an electronic questionnaire to survey the REI applicant pool over a three-year time frame to determine what objective characteristics may increase the likelihood of successfully matching into a REI fellowship position.

\section{Materials And Methods}

Similar investigations have been performed surveying applicants and fellowship directors in other subspecialties [2-6]. These studies were used as guides in constructing the survey used in the current study. A total of 191 applicants participated in the REI Fellowship match between 2011 and 2013. An electronic questionnaire was distributed to 130 applicants whose contact information was available. Follow-up reminders were sent approximately two and four weeks after the initial email. Applicants were asked questions regarding demographic information, educational background, research experience, abstract and publication record, CREOG exam scores, interview invitations, and match outcome. Specific variables collected included age, sex, race, type of medical school (allopathic vs. osteopathic), if graduate degree was obtained, type of obstetrics and gynecology $(\mathrm{OB} / \mathrm{GYN})$ residency training program (academic, university affiliated, or community), if a REI fellowship was available at their OB/GYN residency institution, if elective research block was utilized, years and type of prior research experience, number and first authorship of oral or poster abstract presentations and scientific publications, CREOG exam scores, number of programs applied to, interviews offered and attended, number of programs ranked, position on the rank list of the matched program, if they matched at an institution where they did OB/GYN residency or an elective research rotation, and if they planned to reapply for REI fellowship if the match outcome was unsuccessful.

Statistical analysis was performed with SPSS 22. To determine differences between applicants that matched and did not match, parametric and nonparametric analyses were done after determining if variables met the normality and homoscedasticity assumptions. Mann-Whitney U test was used to compare continuous and discrete ordinal variables, and Chi-square or Fisher's exact test was used to compare nominal variables, where appropriate. A probability less than 0.05 was considered statistically significant.

\section{Results}

Fifty-five applicants (42.3\%) responded to the survey, of which 46 candidates successfully matched (83.6\%). Mean age in our study group was 30.4 years, with more female respondents $(44 / 55 ; 80 \%)$ and with the predominant ethnicity being Caucasian $(28 / 55 ; 51 \%)$. When divided into groups based on match outcome, candidates who matched were younger (30.0 $\pm 0.3 \mathrm{vs.} 32.4$ \pm 1.3 years old, $\mathrm{p}=0.02$ ); otherwise, there were no other significant differences in candidates' demographic background. Ten respondents graduated from a foreign medical institution, of which 70\% (7/10) matched, and the remaining 45 graduated from allopathic medical institutions, of which 85\% (39/45) matched. No applicants from osteopathic medical schools responded to this survey. Nine applicants obtained a graduate degree (2 MS, $3 \mathrm{MPH}$, and $4 \mathrm{PhD}$ ), and eight of the nine matched. Seventy-eight percent of applicants (43/55) completed their OB/GYN residency training at academic institutions, 12.7\% (7/55) from university-affiliated institutions, and 9.1\% (5/55) from community training programs. Having obtained a graduate degree and type of medical school was not significantly associated with match outcome; however, significantly fewer applicants from community OB/GYN training programs matched. Ninety-one percent $(50 / 55)$ of respondents were participating in the match for the first time. Five applicants were participating in the match for the second time. Of the applicants participating in the match for the first time and second time, 84\% (42/55) and 80\% (4/5) 


\section{Cureus}

matched, respectively. There were no significant differences in match outcome based on Alpha Omega Alpha (AOA) or Phi Beta Kappa membership, serving as administrative chief resident, or having an REI fellowship at their home institution (Table 1).

\begin{tabular}{|c|c|c|c|}
\hline & Did Not Match $(n=9)$ & Matched $(n=46)$ & P-val \\
\hline Age (years) & $32.4 \pm 1.3$ & $30.0 \pm 0.3$ & $0.02^{a}$ \\
\hline \multicolumn{4}{|l|}{ Sex } \\
\hline Female & $6(67 \%)$ & $38(83 \%)$ & \multirow{2}{*}{$0.36^{\mathrm{C}}$} \\
\hline Male & $3(33 \%)$ & $8(17 \%)$ & \\
\hline \multicolumn{4}{|l|}{ Race } \\
\hline White & $5(56 \%)$ & $23(50 \%)$ & \multirow{5}{*}{$0.96^{b}$} \\
\hline AA & $2(22 \%)$ & $6(13 \%)$ & \\
\hline Asian & $2(22 \%)$ & $9(20 \%)$ & \\
\hline Hispanic & $0(0 \%)$ & $2(4 \%)$ & \\
\hline Other & $0(0 \%)$ & $6(13 \%)$ & \\
\hline
\end{tabular}

Current OB/GYN resident

\begin{tabular}{|c|c|c|}
\hline No & $3(33 \%)$ & $5(11 \%)$ \\
\hline Yes & $6(67 \%)$ & $41(89 \%)$ \\
\hline
\end{tabular}

Foreign graduate

\begin{tabular}{|c|c|c|}
\hline No & $6(67 \%)$ & $39(85 \%)$ \\
\hline Yes & $3(33 \%)$ & $7(15 \%)$ \\
\hline
\end{tabular}

Graduate degree

$\begin{array}{llcr}\text { No } & 8(89 \%) & 38(83 \%) & 1.00^{\mathrm{C}} \\ \text { Yes } & 1(11 \%) & 8(17 \%) & \end{array}$

AOA

\begin{tabular}{|c|c|c|}
\hline No & 7 (78\%) & $35(76 \%)$ \\
\hline Yes & $2(22 \%)$ & $11(24 \%)$ \\
\hline
\end{tabular}

Phi beta kappa

\begin{tabular}{|c|c|c|}
\hline No & $7(78 \%)$ & $38(83 \%)$ \\
\hline Yes & $2(22 \%)$ & $8(17 \%)$ \\
\hline
\end{tabular}

Administrative chief

No

$5(56 \%)$

$23(50 \%)$ 


\section{Cureus}
Yes
$4(44 \%)$
$23(50 \%)$

OB/GYN residency type

Academic

Community

University-affiliated
$5(56 \%)$

$3(33 \%)$

$1(11 \%)$
$38(83 \%)$

$2(4 \%)$

$0.02^{b}$

$6(13 \%)$

REI fellowship at their OB/GYN residency institution

No

Yes
$6(67 \%)$

$3(33 \%)$
$22(48 \%)$

$24(52 \%)$

\section{TABLE 1: Differences in Demographic/ Educational Background between Matched and Unmatched Candidates}

$\mathrm{AA}=$ African American; $\mathrm{a}=$ Comparison done using Mann-Whitney $\mathrm{U}$ test; $\mathrm{b}=$ Comparison done using Chi-square test; $\mathrm{c}=$ Comparison done using Fisher's Exact test

The majority of applicants had prior research experience $(50.9 \% ; 28 / 55)$, which was defined as dedicated research time without clinical duties excluding elective research blocks during OB/GYN residency. Of those with prior research experience, $20 \%(11 / 55)$ had basic science, 7.3\% (4/55) had clinical, and 23.6\% (13/55) had both prior clinical and basic science research experience. Thirty applicants (54.5\%) had dedicated elective research blocks within their OB/GYN residency programs, and 22 candidates (40\%) pursued away rotations. The number of applicants with prior research experience, elective research blocks, and away rotations were similar between the applicant groups that did and did not match.

Candidates who matched had significantly more first author poster abstract presentations than unmatched applicants did (3.4 \pm 0.4 vs. $1.1 \pm 0.4, \mathrm{p}=0.01)$. Moreover, matched applicants tended to have more publications accepted $(3.3 \pm 0.8$ vs. $2.0 \pm 1.0, p=0.14)$ and more publications submitted ( $1.5 \pm 0.4$ vs. $0.8 \pm 0.4$, p=0.16); however, these findings were not statistically significant. Similarly, there were no significant differences in publication record when first authorship or REI-related subject matter was considered. Other factors, such as oral abstract presentations, CREOG scores, or book chapter publications, were not significantly different between the matched and unmatched groups (Table 2).

\section{Did Not Match $(n=9)$}

Research block

$$
\text { No }
$$

Yes

$5(56 \%)$

Away rotation

No
Matched $(n=46)$

P-value
$28(61 \%)$ 


\section{Cureus}

Yes

Prior research experience

No

Yes

Type of prior research experience

None

Basic science

Clinical

Both

Grant writing experience

No

\# of publications accepted - first author

\# of publications accepted - REI field

\# of publications submitted

\# of publications submitted - first author

\# of publications submitted - REI field

Abstracts (poster)

Abstracts (poster) - first author

Abstracts (poster) - REI field

Abstracts (oral)

Abstracts (oral) - first author

Abstracts (oral) - REI field

Published book chapter

Published book chapter - first author

Published book chapter - REI field

Highest CREOG score

\# of programs applied to
$7(78 \%)$

$2(22 \%)$

$2.0 \pm 1.0$

$0.8 \pm 0.5$

$7(78 \%)$

$20(43 \%)$

$2(22 \%)$

$9(20 \%)$

$4(9 \%)$

$13(28 \%)$

$0(0 \%)$

$0.16^{b}$

$0.9 \pm 0.6$

$0.8 \pm 0.4$

$0.6 \pm 0.3$

$0.6 \pm 0.3$

$2.9 \pm 0.8$

$1.1 \pm 0.4$

$2.0 \pm 0.7$

$1.3 \pm 0.6$

$0.8 \pm 0.4$

$0.9 \pm 0.4$

$0.3 \pm 0.2$

$0.2 \pm 0.1$

$0.3 \pm 0.1$

$0.3 \pm 0.1$

$222.4 \pm 1.8$

$0.16^{\mathrm{a}}$

$32.6 \pm 2.2$

30 (65\%)

16 (35\%)

$3.3 \pm 0.8$

$0.14^{a}$

$1.9 \pm 0.6$

$0.16^{a}$

$1.6 \pm 0.7$

$0.35^{a}$

$1.5 \pm 0.4$

$0.16^{a}$

$1.2 \pm 0.3$

$0.17^{a}$

$0.9 \pm 0.1$

$0.15^{a}$

$4.5 \pm 0.8$

$0.38^{a}$

$3.4 \pm 0.4$

$0.01^{a}$

$2.7 \pm 0.5$

$0.49^{a}$

$1.3 \pm 0.3$

$0.78^{a}$

$1.2 \pm 0.2$

$0.54^{a}$

$0.9 \pm 0.2$

$0.86^{a}$

$0.4 \pm 0.1$

$0.97^{a}$

$0.1 \pm 0.1$

$0.95^{a}$

$216.6 \pm 3.8$

$30.6 \pm 1.4$

$0.79^{a}$ 


\section{Cureus}

\# of programs interviews offered

\# of programs interviews attended

\# of programs ranked
$8.7 \pm 2.1$

$8.1 \pm 1.8$

$8.1 \pm 1.8$

$13.3 \pm 0.7$ $0.002^{\mathrm{a}}$

$0.01^{a}$

$0.01^{\mathrm{a}}$

\section{TABLE 2: Differences in Application between Matched and Unmatched Candidates}

\#= Number; REI= Reproductive Endocrinology and Infertility; CREOG= Council on Resident Education in Obstetrics and Gynecology; $a=$ Comparison done using Mann-Whitney $U$ test; $b=$ Comparison done using Chi-square test; $c=$ Comparison done using Fisher's Exact test

While candidates applied to a similar number of programs $(\mathrm{p}=0.79)$, matched applicants received more interview offers $(17.7 \pm 1.1$ vs. $8.7 \pm 2.1, \mathrm{p}=0.002)$, attended more interviews $(13.7$ \pm 0.7 vs. $8.1 \pm 1.8, \mathrm{p}=0.01)$, and ranked more programs ( $13.3 \pm 0.7$ vs. $8.1 \pm 1.8, \mathrm{p}=0.01)$ (Table 2 ). Twenty-six respondents matched to their top program, and 34 matched to a program in the top three of their rank list. Twelve people matched at an institution where they did OB/GYN residency, research, or an elective away rotation; and for nine individuals, this was their first choice.

Interestingly, five first time applicants without accepted publications did successfully match. Table 3 presents application characteristics of these candidates. Four of these applicants had one to two papers submitted for publication and had one to five poster presentations. Two applicants had published book chapters (as first author in the REI field) and additionally had grant writing experience. All of these applicants were US medical graduates, one had a master degree, and one had over to years of basic science research experience. CREOG scores ranged from 209 to 222. Two of these candidates matched at an institution where they did research or fellowship work.

Did Not Match $(n=5)$

OB/GYN residency type

Academic

$3(60 \%)$

$1(20 \%)$

$1(20 \%)$

University-affiliated

Community

$4(80 \%)$

Yes

$1(20 \%)$

$3(60 \%)$

Away rotation

No

$3(60 \%)$

$3(60 \%)$

Yes

$2(40 \%)$

$3(60 \%)$

$1(20 \%)$

$1(20 \%)$

Matched $(n=5)$

Graduate degree 


\section{Cureus}

No

Yes

$5(100 \%)$

$0(0 \%)$

$4(80 \%)$

$4(80 \%)$

$1(20 \%)$

$1(20 \%)$

Type of prior research experience

None

Basic science

\# of publications submitted

\# of publications submitted - first author

\# of publications submitted - REI field

Abstracts (poster)

Abstracts (poster) - first author

Abstracts (poster) - REI field

Abstracts (oral)

Published book chapter

Highest CREOG score

\# of programs applied to

\# of programs interviews offered

\# of programs interview attended

\# of programs ranked
$4(80 \%)$

$4(80 \%)$

$1(20 \%)$

$1(20 \%)$

$0.2 \pm 0.2$

$1.2 \pm 0.4$

$0.2 \pm 0.2$

$0.6 \pm 0.2$

$0.2 \pm 0.2$

$1.0 \pm 0.3$

$1.2 \pm 0.5$

$2.6 \pm 0.7$

$0.6 \pm 0.4$

$1.8 \pm 0.4$

$0.4 \pm 0.4$

$2.4 \pm 0.7$

$1.0 \pm 1.0$

$0 \pm 0$

$0.4 \pm 0.2$

$0.6 \pm 0.4$

$219.2 \pm 5.0$

$216.6 \pm 2.6$

$32.8 \pm 4.1$

$31.6 \pm 3.6$

$8.0 \pm 2.0$

$15.6 \pm 1.5$

$7.8 \pm 2.1$

$12.6 \pm 0.7$

$7.8 \pm 2.1$

$12.4 \pm 0.5$

\section{TABLE 3: Characteristics of First Time Applicants without Accepted Publications}

\#= Number; REI= Reproductive Endocrinology and Infertility; CREOG= Council on Resident Education in Obstetrics and Gynecology; $a=$ Comparison done using Mann-Whitney $U$ test; $b=$ Comparison done using Chi-square test; $c=$ Comparison done using Fisher's Exact test

Lastly, five applicants were participating in the match for the second time after added research experience or serving as OB/GYN faculty, and four of these candidates matched. Of those who matched, three graduated from academic OB/GYN institutions, and one had an REI fellowship program at their home institution. All had first author poster and oral abstracts, three had publications accepted, and all had publications submitted as first author in the field of REI. All four ultimately matched at their first or second choice program on their rank list, only one of which matched at an institution where they had done research. 


\section{Discussion}

The reproductive endocrinology and infertility match is one of the most competitive subspecialty match processes within the field of OB/GYN. Interestingly, data from the National Resident Matching Program from 2008 to 2011 demonstrated a notable decline of applicants applying to REI of approximately $21.6 \%$ [1]. This trend may be a reflection of how competitive this match process has become, thus deterring some applicants from applying. It is generally believed that important factors for matching in REI are publication record, research experience, and CREOG scores. The extent to which these criteria influence match outcome, however, has not been investigated in the literature. Because this information may be valuable to future $\mathrm{OB} / \mathrm{GYN}$ residents considering applying to REI fellowship and potentially fellowship applicants in other subspecialties as well, we conducted a survey of REI fellowship applicants over a threeyear period to determine what factors may correlate with match outcome.

Previous studies in other subspecialties have shown publication record [3-5] and presentations at national conferences [5] significantly associated with a successful match outcome. Similarly, the current study suggests that research experience is an important contributor to a successful match. Matched candidates had significantly more poster abstracts presented as first author. Additionally, though not statistically significant, matched applicants tended to have more publications accepted and more publications submitted. Interestingly, there was no significant difference between those who did and did not match in terms type of research experience of if the research was conducted within the field of REI. Therefore, demonstrating the ability to present research of scientific merit as first author, regardless of the field of study or specific type of research, seems to be important in the selection process.

The majority of applicants (80\%) had publications accepted at the time of applying to fellowship. Ten first-time candidates, however, had no accepted publications, and five of the 10 applicants did successfully match. Although these individuals did not have publications accepted at the time of applying for REI fellowship, their work ethic and commitment to research were demonstrated through alternative means (for example, book chapter publication, funded grant writing experience, and >two years of basic science research experience) that may have played a role in securing a fellowship position. Three of the five subsequently matched at their top choice institution. These findings suggest that demonstration of dedicated research involvement can increase the likelihood of successful match in candidates without extensive publication record. It is important to note, however, that the sample size in this analysis is too small to draw any meaningful conclusions.

In addition to research, $\mathrm{OB} / \mathrm{GYN}$ residency training at an academic or university-affiliated program may also increase the probability of obtaining a REI fellowship position. Other factors thought to influence match outcome, such as CREOG score, additional graduate degree, having an REI fellowship at their home institution, honor society membership, and serving as administrative chief, did not appear to significantly affect match rates.

Our study is limited by its low response rate and potential selection bias resulting from an unbalanced response rate from matched versus unmatched candidates. Although the survey was anonymous, it is possible that some candidates did not wish to divulge details about their application. The extent to which our respondent pool represents the general REI fellowship applicant is not known, particularly since the majority of individuals that answered our survey had successfully matched. Therefore, the ability to generalize these findings over a large scale of REI fellowship applicants may be limited. Future studies of a larger sample size incorporating a more balanced response rate from matched and unmatched candidates are needed to verify our findings and to further examine factors that might be independently predictive of a successful match outcome. In addition, a follow-up study surveying REI fellowship directors to assess what criteria they deem most valuable in their selection process would be of interest. For 
example, the extent to which interpersonal skills, ability to work diligently and work well with others, and how good a "fit" they felt the applicant was are factors that could be relevant in the match process, but could not be evaluated based on the nature of the current study.

To our knowledge, this is the first study to evaluate applicant characteristics pursing REI fellowship. Presenting poster abstracts as first author was an important factor in matching among the respondents in our survey and publishing scientific articles may also be associated with higher chances of matching. Although the current study has limitations that may restrict the conclusions we are able to draw, our findings may still be useful to future applicants interested in pursuing a career in REI.

\section{Conclusions}

Research experience, particularly presenting poster abstracts as first author, was significantly associated with a successful match into a REI fellowship. There was also a trend for matched applicants to have more publications accepted and submitted at the time of the interview process.

\section{Additional Information \\ Disclosures}

Human subjects: All authors have confirmed that this study did not involve human participants or tissue. Animal subjects: All authors have confirmed that this study did not involve animal subjects or tissue. Conflicts of interest: In compliance with the ICMJE uniform disclosure form, all authors declare the following: Payment/services info: All authors have declared that no financial support was received from any organization for the submitted work. Financial relationships: All authors have declared that they have no financial relationships at present or within the previous three years with any organizations that might have an interest in the submitted work. Other relationships: All authors have declared that there are no other relationships or activities that could appear to have influenced the submitted work.

\section{References}

1. National Resident Matching Program. Results and Data. Matching Service 2013 Appointment Year. (2013). Accessed: February 28, 2014: http://www.nrmp.org/match-data/fellowshipmatch-data/.

2. Harper JG, Given KS, Pettitt B, Losken A: The independent plastic surgery match: An in-depth analysis of the applicants and process. Ann Plast Surg. 2011, 66:568-71.

10.1097/SAP.0b013e31820b3cea

3. Beres A, Baird R, Puligandla PS: Success in the Pediatric Surgery Match: A survey of the 2010 applicant pool. J Pediatr Surg. 2011, 46:957-61. 10.1016/j.jpedsurg.2011.02.030

4. Hirthler MA, Glick PL, Hassett JM Jr, Cooney DR: Evaluation of the pediatric surgical matching program by the directors of pediatric surgical training programs. J Pediatr Surg. 1994, 29:1370-4.

5. Hirthler MA, Glick PL, Hassett JM Jr, Rossman J, Mendola P, Allen JE, Jewett TC Jr, Cooney DR: Comparative analysis of successful and unsuccessful candidates for the pediatric surgical matching program. J Pediatr Surg. 1992, 27:142-5.

6. Muffly TM, Penick ER, Tang F, Bonham AJ, Smith RP, Hill RF, Blandon RE: Factors used by female pelvic medicine and reconstructive surgery fellowship directors to select their fellows. Int Urogynecol J. 2010, 21:349-52. 10.1007/s00192-009-1038-z 